\title{
La convergencia entre el derecho de la competencia y los derechos de propiedad intelectual

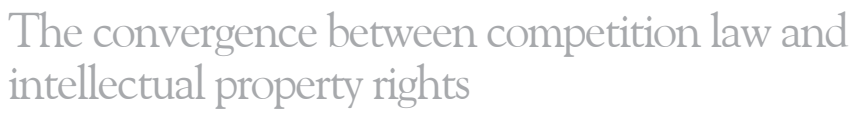

HEBERT EDUARDO TASSANO VELAOCHAGA***

Resumen: El Instituto Nacional de Defensa de la Competencia y de la Protección de la Propiedad Intelectual (INDECOPI) tiene entre sus funciones tanto la defensa de la libre competencia como la protección de la propiedad intelectual. Este diseño institucional tiene la ventaja de permitir apreciar con mayor claridad cuáles son los puntos de convergencia entre ambas materias, armonizarlos y conseguir los objetivos que tienen en común. Dentro de esta convergencia, existen temas sensibles, como el otorgamiento de licencias obligatorias, por lo que en el presente trabajo se destaca su carácter de medida excepcional y se plantea que, para considerar su otorgamiento, el Estado debe contar con un procedimiento que brinde confianza y predictibilidad a la ciudadanía y con definiciones claras sobre qué debemos entender por interés público, emergencia y seguridad nacional. Finalmente, se propone que su otorgamiento tenga justificación en un análisis costo beneficio que arroje como resultado que, en efecto, era la opción más adecuada.

Palabras clave: competencia - propiedad intelectual - patentes - licencias obligatorias - abuso de posición dominante - análisis costo beneficio

Abstract: INDECOPI has within its functions the defense of free competition and the protection of intellectual property. This institutional design has the advantage of being able to see more clearly what are the points of convergence between the two subject-matter, harmonize them and achieve the goals they have in common. Within this convergence, there are sensitive issues as the granting of compulsory licenses. In this work, we highlight that compulsory licenses are exceptional measures and, to consider its granting, the State must have a procedure that provides confidence and predictability to citizens and clear definitions of what is meant by public interest, emergency and national security. Finally, it is proposed that the granting of compulsory licenses should be justified by a cost benefit analysis showing that is the best choice.

Key words: competition - intellectual property - patents - compulsory licensing - abuse of dominant position - cost benefit

* Para la elaboración de este artículo se contó con la colaboración de Patricia Sarria Pardo, asesora de la Presidencia del INDECOPI. Las opiniones vertidas en este artículo representan la visión exclusiva del autor sobre las diferentes materias abordadas, de modo que no comprometen en modo alguno la posición de las instituciones de las cuales es parte el autor.

* Presidente del Consejo Directivo del INDECOPI (Perú). Abogado por la Pontificia Universidad Católica del Perú (PUCP). Máster en Regulación de Servicios Públicos por la Universidad Peruana de Ciencias Aplicadas (Perú) y el IEDE Business School (España), Universidad Europea de Madrid (España). Profesor en la PUCP, ESAN, Universidad del Pacífico y Universidad San Martín de Porres. Correo electrónico: htassano@indecopi.gob.pe 
CONTENIDO: I. INTRODUCCIÓN.- II. RELACIÓN ENTRE EL DERECHO DE LA COMPETENCIA Y LA PROPIEDAD INTELECTUAL.- II.1. SUPUESTO DONDE EL TITULAR DE UN DERECHO DE PROPIEDAD INTELECTUAL ABUSA DE SU POSICIÓN DE DOMINIO. CASOS QUE INVOLUCRAN DERECHOS DE PROPIEDAD INTELECTUAL.- II.2. LAS LICENCIAS OBLIGATORIAS COMO ALTERNATIVA DE REMEDIO.- III. EL ANÁLISIS ECONÓMICO DEL DERECHO PARA EVALUAR LA PERTINENCIA DE LAS MEDIDAS.- IV. REFLEXIONES FINALES.

\section{INTRODUCCIÓN}

A primera vista, podríamos pensar que el derecho de la competencia y el derecho de la propiedad intelectual no guardan relación alguna. Por un lado, el derecho de propiedad intelectual otorga derechos exclusivos como patentes, modelos, diseños industriales, derechos de autor, marcas y otros, que podrían ser equiparables a un monopolio. Por otro lado, la principal función del derecho de la competencia es fomentar que existan muchos competidores en el mercado, promoviendo la competencia como la manera más eficiente de asignación de los recursos, cuyo objetivo primordial es aumentar el bienestar general de la población ${ }^{1}$.

Los derechos de propiedad intelectual resultan actualmente muy importantes para el desarrollo y la competitividad de un país. Por ello, se necesita un sistema de protección de la propiedad intelectual sólido que proteja estos derechos y brinde el soporte necesario para la innovación tecnológica, considerando como premisa básica que el progreso técnico crea un aumento en la competencia en el ámbito de la investigación y el desarrollo. Asimismo, el derecho de la competencia es fundamental para que el mercado funcione de manera eficiente, ya que cuenta con una normativa que permite promover la competencia y corregir las prácticas anticompetitivas que tienen lugar en el mercado. Considerando que ambos derechos son fundamentales para el desarrollo del país, la propiedad intelectual y la promoción y defensa de la competencia deben ser considerados como elementos pares y necesarios de un sistema jurídico y económico dinámico, a pesar de que en algunos puntos de su interacción pueden aparecer algunas controversias aparentes.

La finalidad de este trabajo es conocer algunos de los puntos de interacción entre la competencia y la propiedad intelectual, y, en particular, la convergencia que puede ocurrir entre derechos de propiedad protegidos por patentes de invención y la defensa de la competencia cuando el titular del derecho de patente hace abuso de su posición de dominio para distorsionar la competencia. En esa línea, analizaremos en qué casos es posible otorgar una licencia obligatoria y las cuestiones que deberían ser tomadas en consideración al momento de evaluar su otorgamiento.

1 CABANelLas, Guillermo. Propiedad intelectual y libre competencia. Buenos Aires: 2006 (http://www. wipo.int/edocs/mdocs/lac/es/ompi_jpi_bue_06/ompi_jpi_bue_06_8.pdf). 


\section{RELACIÓN ENTRE EL DERECHO DE LA COMPETENCIA Y LA PROPIEDAD INTELECTUAL}

Es preciso indicar que no existe un criterio uniforme sobre qué tipo de relación se configura entre el derecho de la competencia y el de propiedad intelectual. En efecto, hay diversas opiniones sobre si existe o no conflicto entre ambos derechos, ya que el primero fomenta la competencia y el otro otorga una suerte de monopolio temporal. La teoría con la que nosotros coincidimos señala que no hay conflicto entre las normas de competencia y los derechos de propiedad intelectual. Por el contrario, esta teoría sostiene que ambos son parte del sistema legal y tienen el objetivo común de alcanzar la eficiencia económica y el bienestar de los consumidores. Por ejemplo, esta es la posición de las autoridades estadounidenses que sostienen en The Antitrust Guidelines for the Licensing of Intellectual Property que «la ley de la propiedad intelectual y la de competencia comparten el objetivo común de promover la innovación y mejorar el bienestar de los consumidores» ${ }^{2}$.

En nuestra opinión, en efecto, las leyes de defensa de la competencia y las de protección de la propiedad intelectual comparten el objetivo común de promover las innovaciones y mejorar el bienestar de los consumidores debido a la inclusión del progreso técnico como un proceso esencial de la competencia. Ello nos ha llevado a tener una nueva visión de la relación entre ambas disciplinas, teniendo en cuenta que, si bien pueden existir algunos aparentes conflictos en el corto plazo, en el largo plazo sus objetivos se encuentran en armonía.

Sin embargo, son justamente los conflictos a corto plazo entre ambas disciplinas los que han llamado más la atención en los últimos años, ya que en nuestros días los derechos de propiedad intelectual han adquirido importancia en la economía por el rol que cumplen en el desarrollo del país, así como también lo ha hecho la protección jurídica de la competencia, como mecanismo de asignación e innovación en los mercados de productos y servicios. Estos conflictos son más evidentes en países en desarrollo como el Perú, donde existe gran necesidad de desarrollo e innovación, lo cual origina que exista el deseo de que la tecnología — protegida por derechos de propiedad intelectual— deje de ser objeto de derechos exclusivos y pase tan pronto como sea posible al dominio público. En respuesta a ello, el incentivo natural de los titulares de derechos de propiedad intelectual será retrasar la transferencia de estos derechos al dominio público para obtener mayores ganancias, utilizando algunas veces prácticas anticompetitivas.

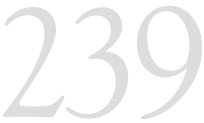

LA CONVERGENCIA ENTRE EL

DERECHO DE LA

COMPETENCIAY

LOS DERECHOS

DE PROPIEDAD

INTELECTUAL

THE

CONVERGENCE

BETWEEN

COMPETITION

LAW AND

INTELLECTUAL

PROPERTY RIGHTS

2 U.S. Department of Justice and the Federal Trade Commission. The Antitrust Guidelines for the Licensing of Intellectual Property, 1995 (http://www.ftc.gov/sites/default/files/attachments/competitionpolicy-guidance/0558.pdf). 
Para entender mejor este punto, veremos a continuación algunos casos en los que el derecho de propiedad intelectual y el derecho de la competencia interactúan, de modo tal que pueden generarse algunos conflictos que la autoridad competente debe resolver de manera adecuada.

El Instituto Nacional de Defensa de la Competencia y de la Protección de la Propiedad Intelectual (INDECOPI) es un organismo público especializado con capacidad jurídica de derecho público interno, que tiene entre sus funciones ser la Agencia Nacional de la Competencia y a la vez la Autoridad de Protección de la Propiedad Intelectual ${ }^{3}$. Este diseño institucional del INDECOPI es único en el mundo, ya que tiene a su cargo incluso otras disciplinas como la protección al consumidor. El hecho de que el INDECOPI tenga a cargo ambas disciplinas permite a la autoridad observar muy de cerca los puntos de interacción que existen y, además, identificar de mejor manera en qué áreas exactamente pueden aparecer conflictos. Otra ventaja importante que aporta esta posición privilegiada es que permite a la autoridad identificar qué políticas públicas pueden ser apropiadas para armonizar el objetivo de promover la innovación y el de defender la competencia con miras a mejorar la eficiencia en el mercado en beneficio de los consumidores.

En efecto, el decreto legislativo 1033 (Ley de Organización y Funciones del INDECOPI) señala que el INDECOPI es un organismo encargado de defender la libre y leal competencia, sancionando las conductas anticompetitivas y desleales y procurando que en los mercados exista una competencia efectiva. Además, es la autoridad competente para administrar el sistema de otorgamiento y protección de los derechos de propiedad intelectual en todas sus manifestaciones. Sin embargo, como hemos adelantado existen áreas de convergencia del derecho de la competencia con la propiedad intelectual que ponen en evidencia algunos conflictos y, por su importancia, merecen ser estudiados.

\section{II.1. Supuesto donde el titular de un derecho de propiedad intelectual abusa de su posición de dominio. Casos que involucran derechos de propiedad intelectual}

Cuando se otorga un derecho de propiedad intelectual, se está otorgando un derecho de exclusividad sobre el uso y explotación del mismo, como, por ejemplo, una patente, una marca, un derecho de autor, entre otros. Por tanto, ante una solicitud de licencia por parte de algún competidor, es legítimo que su titular se niegue a concederla; sin embargo, habrá algunos casos donde esta negativa afecte la libre y leal competencia en el mercado.

3 EI INDECOPI es un organismo público especializado adscrito a la Presidencia del Consejo de Ministros del Perú. Tiene autonomía funcional, técnica, económica, presupuestaria y administrativa. 
Al respecto, el decreto legislativo 1034 (Ley de Represión de Conductas Anticompetitivas) establece sanciones para el abuso de posición de dominio cuando el titular de un derecho de propiedad intelectual se niega a conceder una licencia y, con ello, restringe indebidamente la competencia en el mercado, obteniendo beneficios y perjudicando a los competidores reales. La ley establece la necesidad de demostrar que los efectos restrictivos de la competencia son mayores a los efectos positivos de dicha conducta en el mercado. La autoridad competente para realizar la investigación e imponer una eventual sanción es la Comisión de Defensa de la Libre Competencia del INDECOPI (en adelante, Comisión de Libre Competencia) ${ }^{4}$.

Asimismo, se han presentado y resuelto ante la Comisión de Libre Competencia casos importantes por presuntas prácticas anticompetitivas que han involucrado derechos de propiedad intelectual. Entre los más conocidos tenemos el caso Telecable vs. Telefónica del Perú y Telefónica Multimedia ${ }^{5}$ y el caso Ambev vs. Backus, entre otros ${ }^{6}$. En estos casos en análisis que realizó la autoridad de libre competencia se llevó a cabo en mercados conformados por derechos de propiedad intelectual.

\section{II.2. Las licencias o bligatorias co mo alternativa de remedio}

Esta es el área en la que la relación y posibles conflictos entre los derechos de patentes y el derecho de la competencia se hacen más evidentes. Las licencias obligatorias habilitan a un tercero no titular de una patente a utilizar y explotar un producto patentado sin la autorización de su titular, en casos muy específicos y debidamente determinados por la ley. En efecto, según el Acuerdo sobre los Aspectos de los Derechos de Propiedad Intelectual relacionados con el Comercio (ADPIC) de la Organización Mundial del Comercio (OMC) (en adelante, el Acuerdo),

4 Cabe señalar que, si bien, como hemos visto, las nomas de libre competencia peruanas establecen la facultad de la Comisión de Libre Competencia para sancionar este tipo de práctica anticompetitiva, hasta la fecha no se ha presentado un caso con estas características.

5 En ese caso, la empresa Telecable presentó una denuncia contra Telefónica del Perú y Telefónica Multimedia por presuntas prácticas anticompetitivas en la suscripción de acuerdos exclusivos para la radiodifusión, indicando que dichas empresas se negaron a conceder licencias de propiedad intelectual. El pronunciamiento fue a favor de la denunciante y se ordenó la anulación de todas las cláusulas que implicaban la concesión de transmisión y/o distribución de programación exclusiva. Sin embargo, dos de los cinco miembros del órgano colegiado se opusieron a la decisión de la mayoría, argumentando que el buen funcionamiento de los mercados se basa en la protección de los derechos de propiedad. En su opinión, cualquier limitación de los derechos de propiedad debe estar basada en la creencia de que el bienestar del consumidor se está viendo afectado en el largo plazo y sin justificación. Este caso nos enseña que la concesión de derechos de propiedad intelectual y la transmisión/distribución de la señal pueden limitar la competencia en el mercado. Véase resolución 062-CCO-2000/Osiptel.

6 La empresa Ambev presentó una denuncia contra Backus y otros por abuso de posición dominante, indicando que estaban impidiendo su acceso al sistema de intercambio de envases (SIE) y que Backus y otras empresas minoristas habían celebrado contratos de exclusividad con el fin de impedir el acceso a los principales canales de venta al por menor de cerveza. De manera paralela, Backus solicitó el registro del envase que contenía un símbolo triangular ante la Dirección de Signos Distintivos del INDECOPI como marca tridimensional. En diciembre de 2005, el INDECOPI denegó el registro de la marca tridimensional. Con respecto a la denuncia por presuntas prácticas anticompetitivas, Ambev desistió; sin embargo, se analizó el caso y se concluyó que no se había restringido la competencia, ya que con el impedimento de ingreso al SIE no hubo un impacto en el costo de entrada al mercado por parte de Ambev. Véase resolución 045-2009/CLC-INDECOPI. 
los Estados miembros pueden prever en su legislación excepciones limitadas a los derechos exclusivos del titular de una patente. En virtud de ello, las autoridades nacionales podrán, dentro de los términos que establece el Acuerdo, expedir licencias obligatorias contra la voluntad del propietario de la patente, cuando el interés público lo justifique.

En el artículo 31 literal b) del ADPIC se menciona que solo podrá permitirse el uso de la patente sin la autorización del titular cuando previamente el potencial usuario haya intentado obtener la autorización del titular de los derechos en términos y condiciones comerciales razonables y esos intentos no hayan surtido efecto en un plazo prudencial. Los Estados miembros podrán eximir de esta obligación en caso de emergencia nacional o en otras circunstancias de extrema urgencia, o en los casos de uso público no comercial.

En nuestro país, la norma que aborda el tema corresponde a la Decisión 486 de la Comunidad Andina $(\mathrm{CAN})^{7}$, Régimen Común sobre Propiedad Industrial. Esta norma señala que se puede conceder una licencia obligatoria en los siguientes casos:

- Por falta de uso por más de tres años de concedida la patente o cuatro años desde que se presentó su solicitud de registro. Se indica como condición, además, que la licencia obligatoria solo será otorgada cuando el solicitante haya intentado previamente obtener una licencia contractual del titular de la patente ${ }^{8}$.

- Previa solicitud de un País miembro por la existencia de razones de interés público, de emergencia, o de seguridad nacional y solo durante el tiempo que estas razones permanezcan. Señala, además, que la licencia en cuestión será otorgada por la oficina nacional competente. En el caso peruano, la autoridad recae sobre la Dirección de Invenciones y Nuevas Tecnologías del INDECOPI (en adelante, Dirección de Invenciones), la que deberá establecer el alcance o extensión de la licencia, debiendo especificar el periodo por el que se concede y su objeto.

- De oficio o a petición de parte, la oficina nacional competente puede otorgar licencias obligatorias cuando la autoridad competente en materia de competencia haya determinado que el derechohabiente ha desarrollado conductas de abuso de posición de dominio en el mercado.

7 La CAN es una comunidad de países conformada por Bolivia, Colombia, Ecuador y Perú, unidos voluntariamente para lograr el desarrollo.

8 El tiempo de duración de las patentes debe determinarse de manera adecuada, considerando que, tratándose de un producto sin sustitutos, el costo social se incrementará a medida que la duración de la patente sea mayor. Al respecto, existen países que toman en cuenta esta lógica económica, como Alemania, que requiere que los poseedores de patentes paguen una cuota anual para continuar con ella, la misma que es moderada en los primeros años de vida de la patente, pero luego va aumentando hasta que expira el periodo de duración de la patente. Esto tiene como consecuencia que un buen número de inventos pasan al dominio público antes de la fecha de vencimiento del derecho de patente. 
La normativa Andina también señala siete (7) condiciones que deberán cumplir las licencias obligatorias:

1. No serán exclusivas y no podrán concederse sublicencias.

2. Solo podrán transferirse con la parte de la empresa o de su activo intangible que permite su explotación industrial, debiendo registrarse. Caso contrario, no surtirán efectos.

3. Podrán revocarse cuando las circunstancias que les dieron origen han desaparecido y no es probable que vuelvan a surgir.

4. El alcance y duración se limitarán en función de los fines para los que se concedieron.

5. En el caso de patentes de invención que protegen tecnología de semiconductores, la licencia obligatoria solo se autorizará para uso público o para remediar una práctica declarada contraria a la libre competencia.

6. Contemplará una remuneración adecuada.

7. Los usos deberán estar orientados a abastecer principalmente el mercado interno.

En ese sentido, es claro que se pueden otorgar licencias obligatorias en casos excepcionales, ya sea porque el titular de la patente no la usa por un tiempo determinado, para remediar una práctica declarada contraria a la libre competencia o por razones de interés público, de emergencia o de seguridad nacional. Es decir, si bien las licencias obligatorias, en la práctica, habilitan a un tercero no titular de la patente a utilizar y explotar un producto patentado sin la autorización de su titular, este es un mecanismo que se restringe a casos muy específicos y debidamente determinados por el marco legal. En esa línea, es importante resaltar que otorgar una licencia obligatoria ante el hallazgo de una negativa abusiva a conceder la licencia no tiene como finalidad reducir la protección concedida a las empresas titulares de estos derechos de propiedad intelectual. Por el contrario, la autoridad administrativa únicamente deberá intervenir cuando se demuestre que la negativa a conceder licencias perjudica el bienestar del consumidor a corto y largo plazo.

Sin embargo, este es un tema muy polémico, sobre el cual se ha discutido tanto a favor como en contra. Algunas de las posiciones adoptadas y que deben ser tomadas en consideración cuando se evalúe su otorgamiento son las siguientes:

- Los incentivos de los agentes económicos para innovar podrían disminuir.

- La negativa a otorgar una licencia perjudica el bienestar del consumidor a corto y largo plazo.

\section{LA CONVERGENCIA ENTRE EL \\ DERECHO DE LA \\ COMPETENCIA Y \\ LOS DERECHOS \\ DE PROPIEDAD \\ INTELECTUAL \\ THE \\ CONVERGENCE \\ BETWEEN \\ COMPETITION \\ LAW AND \\ INTELLECTUAL \\ PROPERTY RIGHTS}


- El abuso de posición de dominio se ha realizado a través de un derecho de propiedad legítimamente otorgado o del principio de libertad de contratar?.

Con relación a estas posiciones, debemos enfatizar, nuevamente, que la finalidad de otorgar licencias obligatorias debe ser corregir una práctica anticompetitiva, brindar una solución a la población en caso de interés público, emergencia o seguridad nacional, o permitir que un tercero haga uso de una patente que no está siendo utilizada por su titular por el tiempo establecido en la norma. Es decir, la naturaleza de las licencias obligatorias es mejorar la eficiencia del mercado y, por tanto, que exista mayor innovación y competencia.

\section{EL ANÁLISIS ECONÓMICO DEL DERECHO PARA EVALUAR LA PERTINENCIA DE LAS MEDIDAS}

El análisis económico del derecho es una disciplina que utiliza métodos propios de la economía para pronosticar cómo responderán los individuos ante los cambios de las leyes. En opinión de Robert Cooter y Thomas Ulen, con esta disciplina la economía ofreció una teoría científica para pronosticar los efectos de las sanciones sobre el comportamiento ${ }^{10}$. Para realizar estos pronósticos, es muy importante realizar mediciones sobre la base del análisis costo-beneficio, lo que permitirá identificar a los actores involucrados, así como todos los costos que involucraría otorgar una determinada norma o medida. Los resultados permitirán ponderar los resultados positivos con los negativos y, de esa manera, decidir si resulta pertinente.

El otorgamiento de licencias obligatorias debe realizarse únicamente en las situaciones previstas y teniendo en consideración para el análisis un enfoque de eficiencia y bienestar, para lo cual será de mucha ayuda el análisis costo-beneficio. Este enfoque de eficiencia y bienestar coadyuvará a que las decisiones de intervención pública se encuentren orientadas a la búsqueda de eliminar distorsiones que reducen la eficiencia social, algo que parece más evidente en el caso de otorgamiento de licencias obligatorias por falta de uso de una patente. En efecto, si bien no hemos perdido de vista que estamos hablando de un derecho de propiedad otorgado por la ley, teniendo en cuenta que la innovación es necesaria para el desarrollo del país, en un sentido amplio, la autoridad competente debe contar con un mecanismo que le permita dinamizar el mercado en caso de que algún agente quiera hacer uso directo del producto patentado o explorar la introducción de mejoras sobre la base de dicho producto cuando el producto en cuestión no está

9 EZRACHI, Ariel \& Mariateresa MAgGiolino. European Competition Law, Compulsory Licensing and Innovation. Journal of Competition Law \& Economics, 8, 3 (2012), pp. 595-614.

10 Véase: COOTER, Robert y Thomas ULEN. Derecho y economía. México D.F.: FCE, 1998. 
siendo explotado de manera efectiva por el titular de la patente y los intentos de adquirir sus derechos por la vía contractual han fracasado.

De acuerdo con algunos autores, el mecanismo de licencia obligatoria puede ser de utilidad en el caso de la industria farmacéutica, ya que esta industria está altamente basada en la protección de los sistemas de patentes, pero además se caracterizaría por un régimen regulatorio complicado que haría que estas empresas se involucren en una serie de actividades que buscan extender sus beneficios ${ }^{11}$. Naturalmente, estas actividades pueden generar una elevada sensibilidad social, en la medida en que los productos farmacéuticos involucran la salud pública, siendo precisamente este último elemento una de las razones por las que un gobierno puede valorar favorablemente la concesión de una licencia obligatoria.

Con relación a este punto, hay casos específicos de países de la región donde se han otorgado licencias obligatorias, como podemos ver a continuación:

- Brasil: desde el año 2006, negoció con el laboratorio Merck \& Co. para reducir el precio del antirretroviral Efavirenz, producto que era adquirido por el Estado para el tratamiento del SIDA de casi un millón de brasileños. Ante la negativa del laboratorio para reducir el precio en un 60\%, el presidente Lula Da Silva decidió en 2007 conceder una licencia obligatoria sobre la patente de Merck \& Co. para el producto antirretroviral Efavirenz. Esta licencia obligatoria se justifica dado que tenía lugar un evento de emergencia nacional y necesidad pública, ya que el Estado no contaba con los recursos para financiar el tratamiento de la población (en aumento) con esta enfermedad.

- Ecuador: el presidente Rafael Correa emitió un decreto de interés público general en el año 2009, declarando el acceso a los medicamentos esenciales (más tarde emitió otro decreto en la misma dirección para agroquímicos), estableciendo de esta forma las bases para el otorgamiento de licencias obligatorias en dicho país. En virtud de esta declaración de utilidad pública, determinados importadores de productos genéricos solicitaron la licencia obligatoria para el antirretroviral Ritonavir del laboratorio Abbott. En abril de 2010, se otorgó la primera licencia obligatoria para este medicamento.

No hemos encontrado información sobre si en los Estados Unidos y en los países de la Unión Europea se han emitido licencias obligatorias, ya

11 Véase: CARRIER, Michael A. Competition Law and Enforcement in the Pharmaceutical Industry. En: Ariel Ezraki (ed.). Research Handbook on International Competition Law. Cheltenham:Edward Elgar Publishing, 2012.

LA CONVERGENCIA ENTRE EL

DERECHO DE LA

COMPETENCIAY

LOS DERECHOS

DE PROPIEDAD

INTELECTUAL

THE

CONVERGENCE

BETWEEN

COMPETITION

LAW AND

INTELLECTUAL

PROPERTY RIGHTS 
que dichos países tiene una industria farmacéutica y de biotecnología importante (los laboratorios de investigación principalmente son de los Estados Unidos, Francia, Alemania y Suiza) y no son muy propensos a conceder licencias obligatorias. Sin embargo, dado que estas empresas realizan actividad a nivel internacional y son las titulares de las patentes de los medicamentos, se ven afectadas con las licencias obligatorias otorgadas en otros territorios.

No obstante la experiencia de Brasil y Ecuador emitiendo licencias obligatorias y la exploración adicional que hemos realizado a nivel internacional, no hemos identificado que existan antecedentes sobre qué se debe entender por interés público, emergencia o seguridad nacional. Sin embargo, es muy importante que la autoridad tenga claro, al momento de evaluar la pertinencia de otorgar o no una licencia obligatoria, cuáles son los alcances de dichos términos.

La otra parte del proceso supone que, una vez declarado por parte del gobierno que se someterá una patente a licencia obligatoria, la autoridad encargadade licenciar las patentes deberácontar con un procedimientoque brinde transparencia y seguridad a las partes interesadas. Sobre este punto, podemos citar como ejemplo a Colombia, que ha establecido dentro de su procedimiento que, una vez que el gobierno publica en el diario oficial la declaratoria de razones de emergencia o seguridad nacional para someter a una patente a licencia obligatoria, la Superintendencia de Industria y Comercio deberá publicar en su página web en un plazo determinado lo siguiente: a) que las patentes señaladas en la declaratoria podrán ser licenciadas; b) que se recibirán solicitudes de licencia obligatoria sobre las patentes señaladas en la declaratoria; c) el plazo en que se recibirán las solicitudes; y, d) los términos, condiciones y requisitos especiales que deben reunir las solicitudes de la clase particular de licencia obligatoria.

Como venimos mencionando en este trabajo, el otorgamiento de licencias obligatorias debe realizarse únicamente en las situaciones previstas y teniendo en consideración para el análisis un enfoque de eficiencia y bienestar, para lo cual será de mucha ayuda realizar un análisis costo-beneficio de la medida.

Sibien, como hemos mencionado, una licencia obligatoria solose otorgará en caso de interés público, emergencia o seguridad nacional, antes de otorgarla se debe realizar un análisis técnico sobre la conveniencia de la medida, debiéndose evaluar otras alternativas, a pesar de que nos encontremos ante un caso que cumpla con los supuestos excepcionales que hemos mencionado. Solo si los beneficios superan los costos de la medida se debería otorgar una licencia obligatoria.

El análisis costo-beneficio es de gran utilidad para la toma de decisiones, tanto en el ámbito privado como en el público, así como un mecanismo 
de control. De acuerdo al profesor Sola ${ }^{12}$, para que el Estado realice un correcto análisis costo-beneficio debe llevarse a cabo el siguiente procedimiento:

1. Identificar las opciones posibles, a fin de proceder con su evaluación.

2. Identificar las consecuencias de cada una de las opciones posibles.

3. Asignar un valor a cada uno de los costos. En caso de que sea un factor que no tenga un precio en el mercado, como por ejemplo la salud, las vidas humanas, entre otros, o de que los precios de mercado no representen los verdaderos costos, del mismo modo deberá asignarse un valor a cada factor, a fin de evaluar la pertinencia de la medida.

4. Sumar los costos y los beneficios para determinar cuál de las opciones es la pertinente. La medida elegida deberá arrojar que los beneficios son mayores a los costos.

Es claro que las licencias obligatorias son otorgadas para que el Estado haga frente a una situación de interés público, emergencia y seguridad nacional. Sin embargo, no debemos perder de vista que es una medida excepcional, ya que tiene como consecuencia que el titular de la patente pierda su derecho a usar y explotar un derecho que le fue legítimamente otorgado. Así, se trata de una decisión que tiene consecuencias tanto positivas como negativas que deberán ser ponderadas. Por ejemplo, en el mercado de productos tecnológicos, las empresas invierten grandes sumas de dinero en el desarrollo de sus productos, por lo que, si esta inversión no lleva a obtener beneficios económicos, el empresario no tendría los incentivos suficientes para invertir en innovación, lo que finalmente será perjudicial para el desarrollo y la economía del país. Asimismo, consideramos que debe medirse el impacto económico que una licencia obligatoria puede tener en una empresa, ya que una pérdida considerable podría sacarla del mercado, trayendo como consecuencia que un agente económico ya no realice su actividad y, por tanto, no genere valor, que se genere desempleo y, además, que el Estado ya no perciba ingreso por pago de impuestos.

En ese sentido, antes de otorgar una licencia obligatoria, el Estado deberá evaluar otras alternativas, como proveer directamente el producto necesario para hacer frente a la situación de emergencia o seguridad nacional o implementar medidas de inafectación tributaria. En nuestro país, contamos con un antecedente de inafectación tributaria otorgada mediante la ley 27450, publicada el 19 de mayo de 2001, que exoneraban del pago del impuesto general a las ventas y de los derechos arancelarios a los medicamentos para el tratamiento oncológico y VIH/SIDA, con la

LA CONVERGENCIA ENTRE EL

DERECHO DE LA

COMPETENCIAY

LOS DERECHOS

DE PROPIEDAD

INTELECTUAL

\section{THE}

CONVERGENCE

BETWEEN

COMPETITION

LAW AND

INTELLECTUAL

PROPERTY RIGHTS 
finalidad de reducir el precio de los medicamentos para el tratamiento del cáncer, adquiridos mediante compras públicas.

En definitiva, las licencias obligatorias pueden constituir remedios en los siguientes casos: (i) cuando exista falta de uso de una patente y su titular se niegue a licenciarla a un tercero; (ii) cuando se determine que el titular ha cometido una conducta de abuso de posición de dominio; y, (iii) cuando exista una situación de interés público, emergencia o seguridad nacional. Esta medida debe ser vista como la excepción a la regla y debe contar con un procedimiento debidamente establecido que cree predictibilidad en los administrados. Adicionalmente, para el tercer supuesto, debe estar claramente establecido qué debe entenderse por una situación de interés público, emergencia o seguridad nacional y si la medida es pertinente luego de realizarse un análisis costo beneficio.

\section{REFLEXIONES FINALES}

Existen áreas de convergencia entre el derecho de la competencia y la protección de la propiedad intelectual, lo que no significa que sean derechos que se contrapongan o que se encuentren en conflicto. Como hemos visto, son materias que tienen el objetivo común de incentivar la innovación, lo que originará que la competencia en el mercado sea más eficiente y los consumidores se vean beneficiados. En esa línea, si bien pueden existir algunos conflictos entre ambas materias, como cuando se decide otorgar una licencia obligatoria, debe quedar claramente establecido que el otorgamiento de dichas licencias constituye de una medida de carácter excepcional por falta de uso de una patente y la negativa de su titular a licenciarla a un tercero, cuando se ha detectado que el titular de una patente ha abusado de posición de dominio, o para hacer frente a situaciones donde el interés público, una emergencia o la seguridad pública ameriten una decisión de estas características.

$\mathrm{Al}$ respecto, el INDECOPI tiene una posición privilegiada, pues su diseño institucional le otorga las facultades de ser la Agencia de Competencia y, a la vez, la Autoridad de Propiedad Intelectual. En ese sentido, tiene la ventaja de poder apreciar con mayor claridad cuáles son los puntos de convergencia y de qué manera se puede fortalecer el sistema para que el otorgamiento de licencias obligatorias mantenga su carácter excepcional. Para ello, el Estado debe contar con un procedimiento debidamente establecido que cree predictibilidad en los administrados, con definiciones sobre qué debe entenderse como una situación de interés público, emergencia o seguridad nacional y que determine si la medida es pertinente luego de realizarse un análisis costo beneficio. 